\title{
Outcome analysis of patients presenting with neurological manifestations of Plasmodium Vivax malaria in tertiary care hospital of Mumbai
}

\author{
Salagre K.D ${ }^{1}$, Sahay $\mathbf{R N}^{2}$, Holkar $\mathbf{M}^{3}$ \\ ${ }^{1}$ Dr. Kaustubh Dilip Salagre, Assistant Professor, ${ }^{2}$ Dr. Ravindra Nath Sahay, Associate Professor, ${ }^{3}$ Dr. Maya Holkar, Ex- \\ Postgraduate Student, all authors are affiliated with Department of Medicine, Seth G. S. Medical College \& K. E. M. \\ Hospital, Parel Mumbai, Maharashtra, India.
}

Address for Correspondence: Dr. Kaustubh Dilip Salagre, 1/14, Gomantak CHS Ltd, Mahant Road, Near Utkarsh Mandal, Vile Parle (East), Mumbai. E-mail: kdsalagre@gmail.com

\begin{abstract}
Objective: Plasmodium vivax (P. vivax) infection has been considered for a long time a benign and self-limited disease. Percentage of complicated cases of vivax malaria is on the increasing trend, cerebral malaria being the most dreaded and a potentially life-threatening complication. Material and Methods: A prospective observational study was done after institutional ethics committee approval from July 2011 to February 2012 at tertiary care hospital in Mumbai. We studied the clinical profile and outcome of all the 48 patients above the age of 12 years diagnosed with Cerebral Malaria. Results: Incidence of neurological manifestations of vivax malaria in our hospital was $14.15 \%$, with $32(66.7 \%)$ out of 48 patients being males. The mean age of patients with neurological manifestations of vivax malaria was 29.66 years in discharged patients and 50.88 years in expired patients. Altered sensorium $37(77.1 \%)$ followed by convulsion 28 (58.3\%) were common findings, but focal neurological deficit, Bell's palsy, ataxia, psychosis were also seen. Mortality was higher $(62.5 \%)$ in patients with premorbid conditions ( $\mathrm{p}$ value $<0.05$ ) and that of with other system involvements $(87.5 \%)$ including hepatic, renal, hepatorenal, pulmonary involvement ( $\mathrm{p}$ value $<0.05)$. Association between outcome with older age $\&$ high parasite index patients is significant ( $\mathrm{p}$ value $<0.001$ ), however no such significant association was found in terms of gender distribution. Conclusion: Plasmodium vivax, as has been traditionally believed, is no longer a benign species and is causing presentations akin to $P$. falciparum. It is imperative that clinicians are aware of and ready to handle the complications caused by Plasmodium vivax.
\end{abstract}

Keywords: Plasmodium vivax, Neurological manifestation, Outcome

\section{Introduction}

Plasmodium vivax (P. vivax) infection has been considered for a long time a benign and self-limited disease. However, with implementation of molecular diagnosis, it has become evident that $P$. vivax monoinfection could also be involved in multiple organ dysfunction and severe life-threatening disease as seen in $P$. falciparum infection $[1,2]$. Historically, cases of complicated $P$. vivax malaria have been rare and documented almost exclusively by case reports or small case series. Increasing resistance to chloroquine and

Manuscript received: $6^{\text {th }}$ March 2017

Reviewed: $14^{\text {th }}$ March 2017

Author Corrected: $22^{\text {nd }}$ March 2017

Accepted for Publication: $31^{\text {st }}$ March 2017 commonly used antimalarials in $P$. vivax parasites and the recent reports of involvement of this species in complicated malaria suggests the need for further research in vivax malaria. Neurological manifestations of malaria by convention refers to falciparum malaria, but vivax malaria being more common, the percentage of neurological manifestations of vivax malaria cases is on the increasing trend. Cerebral malaria is the most dreaded and a potentially life-threatening complication. Cerebellar ataxia, extra pyramidal rigidity and various psychiatric symptoms have been described either as the early manifestations of cerebral malaria or as a part of the post malaria neurological syndrome [3]. The 
incidence of malaria in Mumbai is rising because of various factors like overpopulation, lack of cleanliness, construction works, water stagnation, migrant workers, insecticide resistance, and antimalarial drug resistance [4].

This study intend to highlight the increasing incidence of neurological manifestations of vivax malaria and hence of the urgency of diagnosing and treating vivax malaria and not treating it as a benign disease. In the Indian scenario, these patients would initially be treated by the primary and the secondary care physicians. For the same reason, these physicians should be aware of the complications, so that earlier and effective treatment can be initiated and patients who need a referral to a tertiary care can also be identified.

\section{Methodology}

Study Design: A prospective observational study was carried out over 48 subjects after institutional ethics committee approval from July 2011 to February 2012 at tertiary care hospital in Mumbai.

\section{Inclusion Criteria}

- All the patients above the age of 12 years admitted with neurological manifestations with peripheral smear or Optimal positive for Plasmodium vivax.
- Those who are willing to give consent.

\section{Exclusion Criteria}

- Patients with mixed malaria,

- Co-existent leptospirosis and/or dengue.

Detailed history, clinical examination findings were noted and routine hematological, biochemical investigations carried out by treating physician were entered in case record form. We further studied the clinical profile and outcome of these patients.

Statistical method: 'Paired t test' was applied for comparison of variables like age, hemoglobin, platelet count, parasitic index. Chi-square test was used for comparing proportions. The $\mathrm{p}$ value less than 0.05 is considered statistically significant.

Definition: Neurological manifestations of plasmodium vivax malaria by definition refers to presence of high grade fever with altered Sensorium, generalized tonic clonic or partial convulsion when other metabolic \& structural causes have been ruled out, cranial nerve palsy or demylination, ataxia, psychosis, hemiplegia.

\section{Results and Analysis}

Total number of indoor patients of vivax malaria (July 2011-February 2012) in our hospital was 339 out of them 48 patients had neurological manifestations with incidence of $14.15 \%$. 32(66.7\%) out of 48 patients were males and $16(33.3 \%)$ were female. Neurological manifestations in vivax malaria was common in 21-30 years (43.7\%), followed by less than 20 years \& more than 50 years (16.7\% respectively). Maximum cases of neurological manifestations of vivax malaria were found in September (37.5\%) month followed by October (20.8\%). Altered sensorium 37(77.1\%) followed by convulsion $28(58.3 \%$ ) were common finding in neurological manifestations of vivax malaria, but focal neurological deficit, bell's palsy, ataxia, psychosis were also seen (Table1). The co-morbid conditions like hypertension, diabetes mellitus, alcoholism, smoking, ischemic heart disease, observed in patients with neurological manifestations of vivax malaria. Association between pre-morbid conditions \& outcome was statistically significant $(\mathrm{P}<0.05)$. Mortality was increased in patients with premorbid conditions.

Table 1: Neurological manifestations of $P$. Vivax malaria.

\begin{tabular}{|c|c|c|}
\hline Type of Presentation & Frequency & Percent \\
\hline Altered Sensorium & 37 & 77.1 \\
\hline Convulsion & 28 & 58.3 \\
\hline Focal Neurological Deficit & 7 & 14.6 \\
\hline Bell's palsy & 1 & 2.1 \\
\hline Ataxia & 2 & 4.2 \\
\hline Psychosis & 1 & 2.1 \\
\hline
\end{tabular}


Out of 48 patients admitted 40 were discharged and 8 patients succumbed. 5 of expired patients were of age $>50$ years which was statistically significant with $\mathrm{p}$ value of 0.003 .5 out of 8 patients died were male and 3 were female ( $\mathrm{P}$ value 1.00, not significant).33(82.5\%) out of 40 discharged patients had isolated neurological involvement while $7(87.5 \%)$ out of 8 expired patients had neurological with other organ involvement. Association of isolated neurological manifestations of vivax malaria and that of with other system involvements including hepatic, renal, hepatorenal, pulmonary involvement was statistically significant in terms of outcome of disease ( $p$ value $<0.001$ ). Thrombocytopenia was the most common finding in neurological manifestations of vivax malaria. Bleeding due to thrombocytopenia was seen in the form of epistaxis, melena, petechiae, ecchymoses, hematuria, subdural hematoma all necessitating platelet transfusions. The association of thrombocytopenia \& outcome showed all $8(100 \%)$ expired cases \& $35(87.5 \%)$ discharged were having thrombocytopenia. This association however was statistically not significant. Hepatic involvement was seen in $29.2 \%$ and renal failure was seen in $12.5 \%$ of patients with neurological manifestations of vivax malaria.

The association between hepatic involvement \& outcome was statistically significant $(\mathrm{P}<0.037)$, neurological manifestations of vivax malaria with hepatic involvement related mortality was seen in 5 cases out of 8 . The association between renal involvement \& outcome was statistically significant $(\mathrm{P}<0.001)$, neurological manifestations of vivax malaria with renal involvement mortality was seen in $5(62.5 \%)$ cases out of 8 . We found $5(10.4 \%)$ patients with neurological manifestations of vivax malaria with pulmonary involvement, out of which $4(50 \%)$ succumbed to death and association was statistically significant (p value 0.005 ) (Table 2 ).

Table-2: Association between organ involvement \& outcome in P Vivax malaria with neurological involvement $(\mathrm{N}=48)$.

\begin{tabular}{|c|c|c|c|c|c|c|c|c|}
\hline \multirow{2}{*}{ Outcome } & \multirow{2}{*}{} & \multicolumn{2}{|c|}{ Hepatic } & \multicolumn{2}{c|}{ Renal } & \multicolumn{2}{c|}{ Pulmonary } & Total \\
\cline { 3 - 9 } & & Increased & Normal & Increased & Normal & Present & Absent \\
\hline Discharge & Count & 9 & 31 & 1 & 39 & 1 & 39 & 40 \\
\hline & Percent & $22.5 \%$ & $77.5 \%$ & $2.5 \%$ & $97.5 \%$ & $2.5 \%$ & $97.5 \%$ & $100 \%$ \\
\hline Expired & Count & 5 & 3 & 5 & 3 & 4 & 4 & 8 \\
\hline & Percent & $62.5 \%$ & $37.5 \%$ & $62.5 \%$ & $37.5 \%$ & $50.0 \%$ & $50.0 \%$ & $100 \%$ \\
\hline Total & Count & 14 & 34 & 6 & 42 & 5 & 43 & 48 \\
\hline $\begin{array}{c}\text { Fisher's Exact } \\
\text { Test }\end{array}$ & Percent & $29.2 \%$ & $70.8 \%$ & $12.5 \%$ & $87.5 \%$ & $10.4 \%$ & $89.6 \%$ & $100 \%$ \\
\hline
\end{tabular}

Table-3: Age, hemoglobin, platelet count, parasite index comparison in neurological manifestations of vivax malaria.

\begin{tabular}{|c|c|c|c|c|c|}
\hline Group Statistics & Outcome & No. of patients & Mean & $\begin{array}{c}\text { Unpaired T } \\
\text { test }\end{array}$ & P value \\
\hline \multirow[t]{2}{*}{ Age } & Discharge & 40 & 29.66 & 3.96 & 0.00 \\
\hline & Expired & 8 & 50.88 & \multicolumn{2}{|c|}{ Difference is significant } \\
\hline \multirow[t]{2}{*}{ Haemoglobin } & Discharge & 40 & 10.87 & 1.88 & 0.07 \\
\hline & Expired & 8 & 9.10 & \multicolumn{2}{|c|}{ Difference is not significant } \\
\hline \multirow[t]{2}{*}{ Platelets } & Discharge & 40 & $83,500.00$ & 1.54 & 0.13 \\
\hline & Expired & 8 & $52,500.00$ & \multicolumn{2}{|c|}{ Difference is not significant } \\
\hline \multirow[t]{2}{*}{ Parasite index } & Discharge & 40 & 2.43 & 10.56 & 0.00 \\
\hline & Expired & 8 & 11.88 & \multicolumn{2}{|c|}{ Difference is significant } \\
\hline
\end{tabular}


Table-4: Association between parasite index \& outcome in neurlogical manifestations of vivax malaria.

\begin{tabular}{|c|c|c|c|c|}
\hline \multirow{2}{*}{ Parasite Index } & & \multicolumn{2}{|c|}{ Outcome } & \multirow{2}{*}{ Total } \\
\hline & & Discharge & Expired & \\
\hline \multirow{2}{*}{ Less than $5 \%$} & Count & 35 & 1 & 36 \\
\hline & Percent & $97.22 \%$ & $2.77 \%$ & $100.0 \%$ \\
\hline \multirow{2}{*}{5 to $10 \%$} & Count & 5 & 1 & 6 \\
\hline & Percent & $83.3 \%$ & $16.7 \%$ & $100.0 \%$ \\
\hline \multirow{2}{*}{ More than $10 \%$} & Count & 0 & 6 & 6 \\
\hline & Percent & $0.0 \%$ & $100.0 \%$ & $100.0 \%$ \\
\hline \multirow{2}{*}{ Total } & Count & 40 & 8 & 48 \\
\hline & Percent & $83.33 \%$ & $16.66 \%$ & $100.0 \%$ \\
\hline Chi-Square Tests & Value & $\mathrm{df}$ & $\mathrm{P}$ value & \multirow{2}{*}{$\begin{array}{c}\text { Association is } \\
\text { Significant }\end{array}$} \\
\hline Pearson Chi-Square & 31.106 & 2 & 0.000 & \\
\hline
\end{tabular}

We compared neurological manifestations of vivax malaria with respect to variables like age, sex, hemoglobin, parasitic index (PI), and platelet count (Table 3). The mean age of patients with neurological manifestations of vivax malaria was 29.66 years in discharged patients and 50.88 years in expired patients. No significant difference found in hemoglobin and platelets counts but parasite index was higher in expired patients. Thus outcome in older age \& high parasite index patients is poor; however no such significant association was found in terms of gender distribution. Association between parasite index \& outcome is statistically significant, hence high parasite index is suggestive of poor prognosis in patients with neurological manifestations of vivax malaria (Table 4). Association among focal neurological deficit and parasite index is however statistically not significance, hence parasite index is not associated degree of neurological deficit, in vivax malaria.

\section{Discussion}

Neurological manifestations of malaria is usually caused by $P$. falciparum but it has been observed that $P$. vivax malaria, which was otherwise considered to be benign malaria, with a low case-fatality ratio, is now increasingly associated with severe disease, including neurological manifestations, as with P.falciparum malaria. In our prospective observational study, we recruited 48 patients fulfilling the criteria for neurological manifestations of vivax malaria during the study period (July 2011-Feb 2012).

Demographic profile in patients with neurological manifestations of vivax malaria: The age profile of patients admitted with neurological manifestations of vivax malaria in our study depicts that most of the admitted patients $(43.7 \%)$ were in the age group of 21 30 years. The findings observed in our study match with those observed in a retrospective study done in South Canara aimed at studying the demographic profile of malaria. Many of the patients were between the age group of 15 and 40 years, with high incidence between the age group of 21 and 30 years [5]. The factors responsible for the age pattern include outdoor work for young adult males and outdoor sleeping habits which then are more prone to get mosquito bites. Additionally city laborers from outstation stay in shanties with high vector population. The pattern observed in vivax malarial deaths was different as compared to those who survived. The peak of vivax deaths was observed in the age group $>50$ years $(62.5 \%)$. A large multicenter treatment trial conducted in Asia concluded that presenting syndromes in severe malaria depend on age and age is an independent risk factor for a fatal outcome of the disease [6,7]. This explains the higher proportion of mortality observed in older age groups in our study.

Males outnumbered females in number of admissions as well as deaths. The findings in our study are supported by a similar finding highlighting the burden of malaria in India [8]. The deaths are more in men than women across all ages, whereas middle productive ages in general have much higher mortality than children. The reason for male dominance is that male subjects have more outdoor work and more prone to vector bite. 
Clinical profile of patients with neurological manifestations of vivax malaria: Altered sensorium 37 cases $(77.1 \%)$ followed by convulsion 28 cases $(58.3 \%)$ are common manifestations and focal neurological deficit 7 cases $(14.5 \%)$, ataxia 2 cases (4.2\%), bell's palsy 1 case $(2.1 \%)$, psychosis 1 case $(2.1 \%)$ was also found(Table 1$)$. Three cases of Plasmodium vivax malaria (all adult male patients) complicated by seizures and symptoms of diffuse meningoencephalitis were reported by Sarkar et al [9]. Out of 12 cases of cerebral involvement due to vivax malaria reported by Nadkar et al 3 had multiple convulsions; 5 had impaired consciousness and 4 had deep coma [7]. Published reports by various authors have found presentations ranging from seizures, decreased level of consciousness, aphasia, hemiparesis, delirium, coma, stupor, psychosis associated with Plasmodium vivax $[10,11]$.

Outcome analysis of patients with neurological manifestations of vivax malaria: Thrombocytopenia was the most common finding in neurological manifestations of vivax malaria with all expired 8 $(100 \%)$ \& many discharged $35(87.5 \%)$ subjects were having thrombocytopenia. One such study was done in Karachi among 124 patients of Malaria Parasite positive, frequency of thrombocytopenia was $71.87 \%$ $(n=46)$ in falciparum and $93.33 \% \quad(n=56)$ in vivax infection [12]. In another study by Patel $\mathrm{P}$ et al incidence of thrombocytopenia was seen in $83.80 \%$ and $74 \%$ cases of $\mathrm{P}$. falciparum and $\mathrm{P}$. vivax malaria respectively [13]. As shown in Table 2 hepatic involvement was seen in $29.2 \%$ and renal failure was seen in $12.5 \%$ of patients with neurological manifestations of vivax malaria in our study and association was statistically significant in terms of outcome of disease. A report on case series of severe vivax malaria done in Bikaner states that complications observed were hepatic dysfunction and jaundice in 23 $(57.5 \%)$ patients, renal failure in $18(45 \%)$ patients [14].

Thus hepatic dysfunction was the most common complication seen in severe vivax malaria in the study in Bikaner followed by renal failure, which is comparable finding to our study. Another study done in Banaras Hindu University concluded that P. vivax malaria can cause ARF, which occurs more commonly in P. falciparum malaria. The prognosis of ARF in P.vivax malaria is favorable [15]. However in our study we observed than ARF when associated with neurological involvement has poor outcome.
When we studied neurological manifestations of vivax malaria with respect to variables like hemoglobin, parasitic index, and platelet count; no significant difference found in hemoglobin and platelets counts but parasite index was higher in expired patients. Higher parasite index associated with high grade of altered sensorium, while association between parasite index and convulsion or focal neurological deficit is statistically not significant. In study by Kulkarni VK et al PI of $>5 \%$ was observed in 63 patients in falciparum group as compared to 27 patients from vivax group [16]. Only 3 patients with PI of $<5 \%$ died whereas 19 patients with PI of $>5 \%$ died from both the groups. Higher PI was associated with more mortality and this finding was statistically significant.

Limitations of our study: This study was conducted in a tertiary care hospital in a metropolitan city; hence the observation and conclusions of this study cannot be extrapolated to the general population, especially those from rural areas. Also in view of small sample size more studies with larger number of subjects needed to confirm findings. However, this study will help to form the basis for similar studies in the future.

\section{Conclusion}

The highest incidence of neurological manifestations of vivax malaria was seen in males and in the age group of 21-30 years; whereas maximum deaths were seen in the age group of $>50$ years. Mortality was higher $(62.5 \%)$ in patients of neurological manifestations of vivax malaria with premorbid conditions and that of with other system involvements $(87.5 \%)$ including hepatic, renal, hepatorenal, pulmonary involvement and also high PI of $>5 \%$.

Thus, Plasmodium vivax, as has been traditionally believed, is no longer a benign species and is causing presentations akin to $P$. falciparum. It is imperative that clinicians are aware and are ready to handle the complications caused by Plasmodium vivax which have been traditionally associated with $P$. falciparum malaria. The key to management is early diagnosis and initiation of treatment based on a high index of suspicion.

\section{Funding: Nil, Conflict of interest: None Permission of IRB: Yes}

Ethical approval: The study was approved by the Institutional Ethics Committee. 


\section{References}

1. Sina B. Focus on Plasmodium vivax. Trends in parasitology. 2002 Jul 1; 18(7):287-9.

2. Is Plasmodium vivax still a paradigm for uncomplicated malaria? Picot S. Med Mal Infect. 2006 Aug; 36 (8):406-13

3. Garg RK, Karak B, Misra S. Neurological manifestations of malaria: an update. Neurology India. 1999 Apr 1; 47(2):85.

4. Limaye CS, Londhey VA, Nabar ST. The study of complications of vivax malaria in comparison with falciparum malaria in Mumbai. J Assoc Physicians India. 2012 Oct; 60(60):15-8.

5. Muddaiah M, Prakash PS. A study of clinical profile of malaria in a tertiary referral centre in South Canara. Journal of vector borne diseases. 2006 Mar 29; 43 (1):29.

6. Dondorp AM, Lee SJ, Faiz MA, Mishra S,Price R, Tjitra E, Than M, Htut Y, Mohanty S, Yunus EB, Rahman R. The Relationship between age and the manifestations of and mortality associated with severe malaria; Clinical Infectious Diseases 2008 Jul 15: 47(2):151-7.

7. Nadkar MY, Huchche AM, Singh R, Pazare AR. Clinical profile of severe Plasmodium vivax malaria in a tertiary care centre in Mumbai from June 2010January 2011. J Assoc Physicians India. 2012 Oct; 60:11-3.

8. Kumar A, Valecha N, Jain T, Dash AP. Burden of malaria in India: retrospective and prospective view.
The American journal of tropical medicine and hygiene. 2007 Dec 1; 77(6 Suppl):69-78.

9. Sarkar S, Bhattacharya P. Cerebral malaria caused by Plasmodium vivax in adult subjects. Indian Journal of Critical Care Medicine. 2008 Oct 1; 12(4):204.

10. Roman GC, Senanayake N. Neurological manifestation of malaria. Arquivos de neuro-psiquiatria. 1992 Mar; 50(1):03-9.

11. Anderson M. Managemnt of cerebral infection. Journal of Neurology, Neurosurgery \& Psychiatry.1993 Dec 1; 56(12):1243-58.

12. Shaikh QH, Ahmad SM, Abbasi A, Malik SA, Sahito AA, Munir SM. Thrombocytopenia in malaria. J Coll Physicians Surg Pak. 2009 Nov; 19(11):708-10.

13. Patel P, Patel M, Gamit B, Modi J, Kevadiya S, Padsala S. Thrombocytopenia in malaria: Correlation with various prevalent species.2013

14. Kochar DK, Saxena V, Singh N, Kochar SK, Kumar SV, Das A. Plasmodium vivax malaria. Emerg Infect Dis. 2005 Jan 1; 11(1):132-4.

15. Prakash J, Singh AK, Kumar NS, Saxena RK. Acute renal failure in Plasmodium vivax malaria. JournalAssociation of Physicians of India. 2003 Mar 1; 51: 265-7.

16. Kulkarni VK, Agrawal K. A study of clinical profile of malaria with special reference to complications and outcome. Int J Adv Med 2017; 4:317-22.

\section{How to cite this article?}

Salagre K.D, Sahay RN, Holkar M. Outcome analysis of patients presenting with neurological manifestations of Plasmodium Vivax malaria in tertiary care hospital of Mumbai. Int J Med Res Rev 2017;5(03):346-351 doi:10.17511/ijmrr. 2017.i03.21. 